\title{
Microstructure and Nanoindentation of the Rostrum of Curculio longinasus Chittenden, 1927 (Coleoptera: Curculionidae)
}

Sudhanshu S. Singh ${ }^{13 \S}$, Michael A. Jansen ${ }^{2 \S}$, Nico M. Franz ${ }^{2}$, and Nikhilesh Chawla ${ }^{1}$ *

\footnotetext{
${ }^{1}$ Materials Science and Engineering, Arizona State University, Tempe, AZ 85287-6106, USA

${ }^{2}$ School of Life Sciences, Arizona State University, Tempe, AZ 85287-4501, USA

${ }^{3}$ Department of Materials Science and Engineering, Indian Institute of Technology Kanpur, Uttar

Pradesh 208016, India
}

Revised Version Submitted to:

Materials Characterization

May, 2016

*Corresponding author: nchawla@ asu.edu, 480-965-2402

${ }^{\S}$ Both authors contributed equally to this work 


\begin{abstract}
The rostrum is an extension of the cuticle of the head of weevils (Coleoptera: Curculionidae) and is often used to bore holes for oviposition (the process of laying eggs) into host plant tissue where larval development occurs. In members of the genus Curculio Linnaeus, 1758, the rostrum is long, slender, and strongly curved, but is nevertheless used to excavate straight bore-holes in the fruit of various host plants, through significant deformation of this structure. In this study, scanning electron microscopy (SEM) was used to examine the rostrum of Curculio longinasus Chittenden, 1927, leading to a microstructural model that describes its deformation behavior. Specifically, we used the continuous stiffness measurement (CSM) technique in nanoindentation to measure the Young's modulus and hardness of rostrum. The values of Young's modulus and hardness for the endocuticle were measured to be $8.91 \pm 0.93 \mathrm{GPa}$ and $558 \pm 60 \mathrm{MPa}$, respectively. These results are critical for generating accurate finite element models of the head's mechanical behavior while it undergoes deformation.
\end{abstract}

Keywords: Curculio longinasus, Rostrum, Nanoindentation, Young's modulus, Hardness 


\section{Introduction}

The insect cuticle (exoskeleton) has gained tremendous attention in recent years in the materials science domain due to its high strength-to-weight ratio and wide range of Young's modulus spanning over eight orders of magnitude [1]. The cuticle generally has a fibrous composite structure, similar to that of a fiber-reinforced polymer matrix composite, and provides structural support, water loss reduction, and protection from parasites and disease for the insect organism. In general, the cuticle is comprised of three layers: exocuticle, mesocuticle, and endocuticle, with the endocuticle often constituting the thickest region [2].

In many arthropods, including most insects, the regions of cuticle are confluent, and do not always have a distinct or sharp boundary between regions. This lack of discrete boundaries is due to the way in which chitin microfibers are arranged in the cuticle; namely, as a laminate composite. The cuticle is made up of laminae, which are sheets of unidirectional chitin microfibers embedded in a protein matrix [3-5]. These laminae are secreted by a basal epithelial layer parallel to the surface; however, each lamina is deposited so that the in-plane fiber orientation changes slightly in successive layers [6]. This arrangement is sometimes referred to as a "helicoidal ply" or as the Bouligand structure [7]. The exocuticle usually exhibits highly compact spacing between laminae and a greater degree of sclerotization (tanning) than in the other regions of the cuticle, and is therefore generally the stiffest layer [1,2]. The mesocuticle is less compact, and not heavily sclerotized, while the endocuticle is the least compact and least tanned section of the cuticle [8]. Joining these regions there are transitional zones displaying levels of compactness and sclerotization in-between those of the surrounding regions, rather than discrete borders $[2,4]$. 
Beetles (order Coleoptera), have a unique cuticular microarchitecture characterized by a strongly differentiated endocuticle $[9,10]$. While the exocuticle and mesocuticle display the more "typical" Bouligand structure, the endocuticle is arranged in large, unidirectional bundles of chitin named macrofibers or balken [11]. These macrofibers are themselves arranged in unidirectional layers, with the layers set parallel to the surface. As with the laminae of the Bouligand structure, the in-plane fiber orientation angle of each layer changes consistently between layers, and is thought to be the product of the circadian rhythm of the beetles [9]. For weevils, this angle can vary from $30-90^{\circ}$, depending on the location in the body and species in question $[10,12,13]$.

The insect species examined in this study is a member of the beetle family Curculionidae, commonly known as weevils. A defining weevil feature of this group is the rostrum, i.e., an extension of the cuticle of the head which bears the chewing (mandibulate) mouthparts. The rostrum is a rigid structure in the sense that it has no articulations or joints. Many weevils, including the herein examined species Curculio longinasus Chittenden, 1927, use the rostrum to prepare sites for oviposition (the process of laying eggs). The larvae are legless and most frequently develop inside the tissue of their respective host plants [14]. The family Curculionidae is tremendously diverse, with over 50,000 species described. However, there may be as many as 200,000 species in existence, the vast majority of which are therefore undescribed [15].

The biology of Curculio longinasus, as well as its congeners in North America, is described in a monographic revision of the genus Curculio Linnaeus 1758 by Gibson [16, 17]. Accordingly, the distribution of the North America Curculio species ranges from "eastern Texas, west to southeastern Arizona, south into Mexico". The only confirmed host plant for $C$. longinasus is Quercus falcata Michaux, 1801 [16, 18]. As with other species in the genus, 
female $C$. longinasus use their rostrum to excavate a hole into the kernel of an acorn, into which they then deposit an egg [16]. The acorn thus serves as a food source and habitat for the developing larva. When mature, the larva will exit the acorn and burrow into the soil where it will pupate. The adult usually emerges $2-3$ weeks thereafter. During the preparation of sites for oviposition, the rostrum can undergo very significant deformation along multiple axes, as the curved structure bores a straight drilling hole into the host plant fruit (nut) tissue [19]. The mechanical properties of this structure are therefore of great interest both from a materials science perspective and for studying the evolution of this genus.

Nanoindentation has emerged as an excellent technique to obtain mechanical properties of materials at small length scales $[20,21]$. Using this technique, one can measure the material's hardness, Young's modulus, and indentation fracture toughness, via sharp indentation of the corresponding surface. For instance, nanoindentation has been widely used to characterize the mechanical properties of the constituent phases in metallic alloys [22, 23]. Recently, this technique has gained popularity for describing biomaterials and many studies have targeted the cuticle of insects [2, 24-29]. Most studies have been performed on the outermost exocuticle, where nanoindentation can be performed in the normal direction. However, few studies have reported on the mechanical properties of endocuticle [2, 28].

Here we report on a successful nanoidentation approach and analysis for the rostrum of C. longinasus, which is used to develop an accurate microstructural model of the rostrum's mechanical behavior. Nanoindentation was performed on the rostrum to quantify the Young's modulus and hardness of the endocuticle and assess whether or not it is more compliant than that of other beetles. Knowledge of the microstructure and mechanical properties of the rostrum will allow us to better understand its overall mechanical performance during the preparation of sites 
for oviposition, and to more accurately model their behavior. Furthermore, it provides an interesting basis to mimic the structure of the insect in synthetic materials.

\section{Experimental Procedure}

Live specimens of $C$. longinasus were collected into $95 \%$ ethanol for preservation. The specimens were collected on August 12, 2014, near the Southwestern Research Station in the Coronado National Forest, Chiricahua Mountains, Arizona, at a site known as the "Onion Saddle" (31.9334200, -109.2634000), from southern red oak (Quercus falcata Micheaux). A female specimen was selected (Fig. 1a) and the rostrum was removed with a fine-edged razor blade (Fig. 1b). The apical $1 / 4$ of the rostrum was also removed and then discarded. The remaining portion of the rostrum and head capsule were then embedded in EMbed 812. Subsequently, the cuticle was immersed in acetone for 24 hours, and then transferred to a 2:1 mixture of acetone to epoxy resin. The sample remained at $21^{\circ} \mathrm{C}$ for 12 hours on a shaker table (to prevent hardening). It was then placed into a 1:1 mixture of acetone to resin, following by a 1:2 mixture (each for 12 hours and at $21^{\circ} \mathrm{C}$ on a shaker table), before finally being placed into a silicone mold with pure resin. The mold was placed into an oven heated to $38^{\circ} \mathrm{C}$, and the resin was allowed to cure for 24 hours. The resulting block was machined to prepare the apical surface of the sample for microtomy. A Leica Ultracut R Microtome and diamond-knife were used to expose a cross-section (transverse plane) of the apical and basal portions of the rostrum and to remove excess material. Semi-thin sections $(0.5 \mu \mathrm{m}$ thick) were kept and stained with toluidineblue-borax. The block was then machined on the opposite side to make the block faces approximately parallel for nanoindentation. The green line in Fig. 1b shows the approximate position on the rostrum where nanoindentation was performed. 
Nanoindentation was conducted on the transverse place using a commercial nanoindenter (Nanoindenter XP-II, Agilent). The block was mounted on an aluminum stub for nanoindentation using superglue. The nanoindenter was first calibrated by measuring Young's modulus and hardness of a silica standard. To minimize the effect of thermal drift, the tests were conducted only when the thermal drift value was lower than $0.05 \mathrm{~nm} / \mathrm{s}$. Experiments were conducted in strain rate control with a strain rate target of $0.05 \mathrm{~s}^{-1}$. A continuous stiffness measurement (CSM) technique was used during indentation [30]. Nanoindentation was carried out using a Berkovich tip on at least 25 locations to a depth of $600 \mathrm{~nm}$. Young's modulus and hardness for an individual indentation were measured as the average value over a depth range, where both modulus and hardness were independent of depth (plateau region). After indentations, the block was sputtered with Au-Pd to obtain a thin coating $(\sim 8 \mathrm{~nm})$ and then SEM was used to analyze the indentations and macrofiber orientation in the rostral apex and rostral base immediately anteriad of the head capsule.

\section{Results and Discussion}

The microstructure of the cross-section (transverse plane) of the rostral apex is shown in Figure 2a. The endocuticle and exocuticle of the rostrum are clearly visible in the magnified subregion shown in Fig. 2b. Cracks induced by the cutting process from microtome/knife are also visible. A less compact helicoidal ply positioned between the endocuticle and exocuticle is visible in Fig. 2c, putatively considered mesocuticle. Most of the rostrum's volume is constituted by the endocuticle, with a very small contribution from the exocuticle (width is $\sim 2-2.5 \mu \mathrm{m}$ ).

Upon close inspection of the endocuticle in the SEM image of the rostrum, it appears that it is made of chitin microfibrils and macrofibers, as shown in Fig. 2b. The endocuticle of $C$. longinasus exhibits a typical "balken" type microstructure [10], with orthogonally stacked layers 
of parallel chitin macrofibers. The macrofibers are bundles of cross-linked chitin microfibrils that are oriented unidirectionally along the longitudinal axis of the bundle, and are unique to beetles [10]. Because the cross-sectional areas of the macrofibers are similar, we can infer (1) that the succeeding layers are stacked at or near $90^{\circ}$ with respect to the longitudinal axes of the macrofibers, and (2) that they are aligned at or near $45^{\circ}$ to the longitudinal axis of the rostrum. On a cylindrical surface, this arrangement would manifest itself as a "ply" of nested, serially orthogonal, $45^{\circ}$ helices. Between the macrofibers and the exocuticle there also appears to be a layer of "lamellate" type cuticle (this could be referred to as "mesocuticle"), with a helicoidal ply comprised of layers of parallel microfibers that are successively rotated about the radial axis of the rostrum [2]. This layer appears to thicken laterally, and tapers off dorsoventrally. A schematization of the observed/inferred structure of the rostrum of $C$. longinasus is shown in Fig. 3.

The base of the rostrum has a more "typical" arrangement of the macrofibers, as seen in Fig. 4, where the stacking angle between each layer results in some fibers being sliced obliquely by the diamond knife. The exocuticle appears smooth, which is likely a result of the highly compact spacing and tanning of the laminae; fewer fibers become dislodged by the knife due to their increased bonding with the matrix, resulting in a smoother cut. A less compact helicoidal ply is not observed. The laminae can only be observed at high magnification, such as in Fig. 5. Additionally, the base of the rostrum (immediately anteriad of the head capsule) exhibits a much thicker proportion of exocuticle, as can be seen in Fig. 6. The fact that the rostrum cuticle has a thinner exocuticle and unique arrangement of macrofibers, compared to the base of the head, suggests that rostral flexibility may arise from alteration of the arrangement of its structural components rather than alteration of their mechanical properties. 
Indentations were made on the layers of endocuticle that were smoothly cut by the diamond knife on both the left and right sides of the rostrum, in the normal direction. No attempt was made to perform indentations on exocuticle, since it was very difficult to locate the exocuticle in the optical microscope (equipped with the nanoindenter) due to the small size of this phase. Moreover, owing to its small width, there would be significant contribution from the surroundings during indentation, especially from the free surface, leading to potentially significant errors in measurement. Similar problems have been reported by Klocke et al. [2] while indenting in the transverse direction. The CSM (continuous stiffness measurement) mode was used instead of the conventional Oliver and Pharr's method. With the CSM technique, a load is applied to the indenter tip to drive the indenter into the specimen surface while concurrently superimposing an oscillating force with a small amplitude (significantly smaller than the nominal load). Using a frequency-specific amplifier, an accurate measurement of contact stiffness at all indentation depths is provided by separating the in-phase and out-of-phase components of the load-displacement data $[20,30]$. The advantage of CSM over the Oliver and Pharr's method is that the modulus and hardness of the material can be obtained with depth on loading. Therefore, multiple indentations are not required. The contact stiffness is calculated using the following equation:

$$
S=\left\lfloor\left(\frac{1}{\frac{F_{o}}{Z_{o}} \cos \phi-\left(K_{s}-m \omega^{2}\right)}\right)-\left(\frac{1}{K_{f}}\right)\right\rfloor^{-1}
$$

Where $F_{0}$ is the force amplitude, $Z_{0}$ is the displacement amplitude, $\omega$ is the frequency of oscillation, $\Phi$ is the phase angle between displacement and the force, $\mathrm{K}_{\mathrm{f}}$ is the frame stiffness, $\mathrm{K}_{\mathrm{s}}$ 
is the spring constant of leaf springs supporting the indenter, and $\mathrm{m}$ is the mass of the indenter. The values of the force amplitude, displacement amplitude, and the phase angle are continuously measured and allow calculating the contact stiffness. From the contact stiffness values, the reduced modulus and the Young's modulus can be calculated using the following equations:

$$
\begin{gathered}
\mathrm{E}_{\mathrm{r}}=\frac{\sqrt{\pi}}{2 \beta} \frac{\mathrm{S}}{\sqrt{A}} \\
\frac{1}{\mathrm{E}_{\mathrm{r}}}=\frac{1-v^{2}}{\mathrm{E}}+\frac{1-v_{\mathrm{i}}^{2}}{\mathrm{E}_{\mathrm{i}}}
\end{gathered}
$$

Where $\mathrm{E}$ and $v$ are Young's modulus and Poisson's ratio of the material, and $\mathrm{E}_{\mathrm{i}}$ and $v_{\mathrm{i}}$ are for the indenter; moreover, $\mathrm{A}$ is the contact area and $\beta$ is the indenter geometry factor. Since the Poisson's ratio of the endocuticle is unknown, a value of 0.3 was taken. In prior analyses of insect cuticle indentation, the reduced modulus have been reported since the Poisson's ratio for the cuticle is unknown. Therefore, in this study the reduced modulus is also reported along with Young's modulus. Hardness $(\mathrm{H})$ can be calculated as $\mathrm{H}=\mathrm{P} / \mathrm{A}$, where $\mathrm{P}$ is the applied load.

Fig. 7 shows two representative curves obtained from nanoindentation. The maximum load for indentation was $\sim 2.5-3 \mathrm{mN}$. The Young's modulus and hardness were observed to be constant with respect to depth (plateau region) between approximately 200-300 $\mathrm{nm}$. Therefore, the Young's modulus and hardness values were taken as the average values between 200-300 $\mathrm{nm}$. Very high values at lower depth of indentation might be attributed to the rounding of the tip and the sample surface roughness. At higher depth, a gradual decrease in the values of Young's modulus and hardness may be attributed to the effect of the surroundings, i.e., the presence of cracks. A summary of the obtained values is provided in Table 1. Our results pertaining to the 
endocuticle also corroborate those of others measured in dry condition [2] indicating that the properties of the rostrum of C. longinasus are similar to those of other insects.

It is well known that the mechanical properties of the insect cuticle are affected by the presence of water. The values of modulus and hardness can decrease significantly in wet conditions compared to dry conditions [2]. To account for this discrepancy, we attempted to perform nanoindentation experiments to observe the effect of water. The block was placed inside water for about 30 minutes and then nanoindentation was performed as soon as the sample was removed from the water. However, the values of modulus and hardness measured in this case were similar to that measured in dry condition. By using in situ nanoindentation, Klocke et al. [2] have shown that the values of modulus and hardness can change within a few minutes of addition and removal of water. In our case, it appears that the water was able to evaporate from the sample during the time the nanoindenter was put for thermal stabilization (required to obtain accurate values at smaller depths), and prior to performing the indentation.

The SEM images of series of indentations made on the endocuticle are shown in Figs. 8a and $8 \mathrm{~b}$; the latter is the magnified version of the area marked in Fig. 8a, showing impressions of three indentations. Only those indentations were taken into account for final calculations, which lie completely inside the chitin macrofibers and are spatially removed from the cracks induced from the microtome process.

\section{Conclusions}

The microstructure and mechanical properties of a rostrum of C. longinasus were quantified using scanning electron microscope and nanoindentation, respectively. A microstructural model of the rostrum, consisting of endocuticle, helicoidal ply and exocuticle, was proposed based on the SEM image analysis. The endocuticle of C. longinasus exhibits a 
typical "balken" type microstructure, with orthogonally stacked layers of parallel chitin macrofibers. Continuous stiffness measurement was used to accurately measure the Young's modulus and hardness values for the endocuticle in the rostrum, with values of $8.9 \pm 0.9 \mathrm{GPa}$ and $558 \pm 60 \mathrm{MPa}$, respectively. These values are fairly typical for dry beetle endocuticle [2]. We therefore believe that the stacking angle of the macrofibers and reduced proportion of exocuticle, rather than an unusually compliant endocuticle, are responsible for the flexibility of the rostrum.

\section{Acknowledgments}

The authors are grateful for financial support from the Office of Naval Research (ONR) under Contract No. N00014-10-1-0350 (Dr. A. K. Vasudevan, Program Manager). The weevil research of Jansen and Franz was funded in part by the National Science Foundation (DEB-1155984) and the United States Department of Agriculture - Agricultural Research Service (Agreement 581275-1-335). The authors acknowledge the use of FIB-SEM facilities within the Leroy Eyring Center for Solid State Science at Arizona State University.

\section{References}

[1] Vincent JF, Wegst UG. Design and mechanical properties of insect cuticle. Arthropod Struct Dev 2004;33:187-99.

[2] Klocke D, Schmitz H. Water as a major modulator of the mechanical properties of insect cuticle. Acta Biomater 2011;7:2935-42.

[3] Bouligand Y. Twisted fibrous arrangements in biological materials and cholesteric mesophases. Tissue Cell 1972;4:189-17.

[4] Neville AC. Biology of the arthropod cuticle, Berlin: Springer Science \& Business Media. 1975. 
[5] Blackwell J, Weih M. Structure of chitin-protein complexes: ovipositor of the ichneumon fly Megarhyssa. J Mol Biol 1980;137:49-60.

[6] Neville AC, Parry DA, Woodhead-Galloway J. The chitin crystallite in arthropod cuticle. J Cell Sci 1976;21:73-82.

[7] Giraud-Guille MM, Chanzy H, Vuong R. Chitin crystals in arthropod cuticles revealed by diffraction contrast transmission electron microscopy. J Struct Biol 1990;103:232-40.

[8] Cheng L, Wang L, Karlsson AM. Mechanics-based analysis of selected features of the exoskeletal microstructure of Popillia japonica. J Mater Res 2009;24:3253-67.

[9] van de Kamp T, Greven H. On the architecture of beetle elytra, Entomol Heute 2010;22:19104.

[10] van de Kamp T, Riedel A, Greven H. Arthropod Structure \& Development Micromorphology of the elytral cuticle of beetles, with an emphasis on weevils (Coleoptera $\square$ : Curculionoidea). Arthropod Struct Dev 2015;45:14-22.

[11] Hepburn HR, Ball A. On the structure and mechanical properties of beetle shells. J Mater Sci 1973;8:618-23.

[12] Leopold RA, Newman SM, Helgeson G. A comparison of cuticle deposition during the preand posteclosion stages of the adult weevil, Anthonomus grandis Boheman (Coleoptera $\square$ : Curculionidae). Int J Insect Morphol 1992;21:37-62.

[13] Davis SR. Morphology, phylogeny, and evolutionary development in the weevils (Insecta: Coleoptera: Curculionoidea). University of Kansas. 2014.

[14] Caldara R, Franz NM, Oberprieler RG. Curculioninae Latreille, 1802. In: Leschen RAB, Beutel RG, editors. Handbook of Zoology. Arthropoda: Insecta: Coleoptera. Volume 3: Morphology and Systematics (Phytophaga), Berlin, Germany: De Gruyter; 2014, p. 305-344.

[15] Oberprieler RG, Anderson RS, Marvaldi AE. Curculionoidea Latreille, 1802: introduction, phylogeny. In: Leschen RAB, Beutel RG, editors. Handbook of Zoology. Arthropoda: Insecta: Coleoptera, Beetles. Volume 3: Morphology and Systematics (Phytophaga), Berlin, Germany: De Gruyter; 2014, p. 285-301.

[16] Gibson LP. Monograph of the genus Curculio in the New World (Coleoptera:

Curculionidae). Part I. United States and Canada. Misc Publs Ent Soc Am 1969; 239-85.

[17] Pelsue FW, O'Brien CW. A redefinition of the Curculionini of the world, with a key to subtribes and genera, and two new genera: Pseudoculio and Megaoculis (Coleoptera: Curculionidae: Curculioninae). Zootaxa 2011;3102:27-49.

[18] Landrum L. Fagaceae: Oak family. J Arizona-Nevada Academy of Sci 1993;27:203-14. 
[19] Toju H, Sota T. Imbalance of predator and prey armament: geographic clines in phenotypic interface and natural selection. The American Naturalist 2006;167:105-17.

[20] Li X, Bhushan B. A review of nanoindentation continuous stiffness measurement technique and its applications. Mater Charact 2002;48:11-36.

[21] Oliver WC, Pharr GM. Measurement of hardness and elastic modulus by instrumented indentation: Advances in understanding and refinements to methodology. J Mater Res 2004;19:3-20.

[22] Singh SS, Schwartzstein C, Williams JJ, Xiao X, De Carlo F, Chawla N. 3D microstructural characterization and mechanical properties of constituent particles in Al 7075 alloys using X-ray synchrotron tomography and nanoindentation. J. Alloys Compd 2014;602:163-74.

[23] Dudek MA, Chawla N. Nanoindentation of rare earth-Sn intermetallics in Pb-free solders. Intermetallics 2010;18:1016-20.

[24] Sun J, Ling M, Wang Y, Chen D, Zhang S, Tong J, Wang S. Quasi-static and dynamic nanoindentation of some selected biomaterials. J Bionic Eng 2014;11:144-50.

[25] Sun J, Tong J, Ma Y. Nanomechanical behaviours of cuticle of three kinds of beetle. J Bionic Eng 2008;5:152-57.

[26] Dai Z, Yang Z. Macro-/micro-structures of elytra, mechanical properties of the biomaterial and the coupling strength between elytra in beetles. J Bionic Eng 2010;7:6-12.

[27] Hayot CM, Enders S, Zera A, Turner JA. Nanoindentation to quantify the effect of insect dimorphism on the mechanical properties of insect rubberlike cuticle. J Mater Res 2013;28:2650-59.

[28] Muller M, Olek M, Giersig M, Schmitz H. Micromechanical properties of consecutive layers in specialized insect cuticle: the gula of Pachnoda marginata (Coleoptera, Scarabaeidae) and the infrared sensilla of Melanophila acuminata (Coleoptera, Buprestidae). J Exp Biol 2008;211:2576-83.

[29] Enders S, Barbakadse N, Gorb SN, Arzt E. Exploring biological surfaces by Nanoindentation. J Mater Res 2004;19:880-87.

[30] Hay J, Agee P, Herbert E. Continuous stiffness measurement during instrumented indentation testing. Exp Tech 2010;34:86-94. 


\section{List of Tables}

Table 1. Summary of the modulus and hardness values of endocuticle in rostrum of Curculio longinasus. 


\section{List of Figures}

Fig. 1. (a) Lateral habitus photograph of the female of Curculio longinasus Chittenden, 1927, (b) lateral view of head of female $C$. longinasus; green bar indicates approximate location of transverse plane used for nanoindentation.

Fig. 2. SEM images of rostrum apex (a) in apical cross-section; (b) magnified view of red region in (a), showing endocuticle, exocuticle, protein matrix, and chitin macrofibers; (c) magnified view of blue region, showing the region of helicoidal ply putatively considered to be mesocuticle.

Fig. 3. Observed/inferred microstructural model for the rostrum of female $C$. longinasus weevils.

Fig. 4. SEM image of rostrum base showing transversely and obliquely sectioned fibers and boundary between exocuticle and endocuticle.

Fig. 5. SEM image of rostrum base at high magnification showing boundary between exocuticle and endocuticle with laminae of exocuticle visible as striations.

Fig. 6. Light microscopy of stained semi-thin sections of rostrum base and apex showing relative proportions of exocuticle and endocuticle.

Fig. 7. Results of the nanoindentation process, applied to the endocuticle of the female $C$. longinasus rostrum; (a) representative load-displacement curves, (b) representative modulus, and (c) hardness curves. Young's modulus and hardness values were averaged between 200-300 nm.

Fig. 8. (a) SEM image of female $C$. longinasus rostrum apex at low magnification, showing multiple indentations (indicated with arrows); (b) magnified SEM image of the region inside red rectangle. Images taken at an angle of $40^{\circ}$. 
Table 1.

\begin{tabular}{|c|c|c|}
\hline Young's Modulus (GPa) & Reduced Modulus (GPa) & Hardness (MPa) \\
\hline $8.91 \pm 0.93$ & $9.71 \pm 1.02$ & $558 \pm 60$ \\
\hline
\end{tabular}



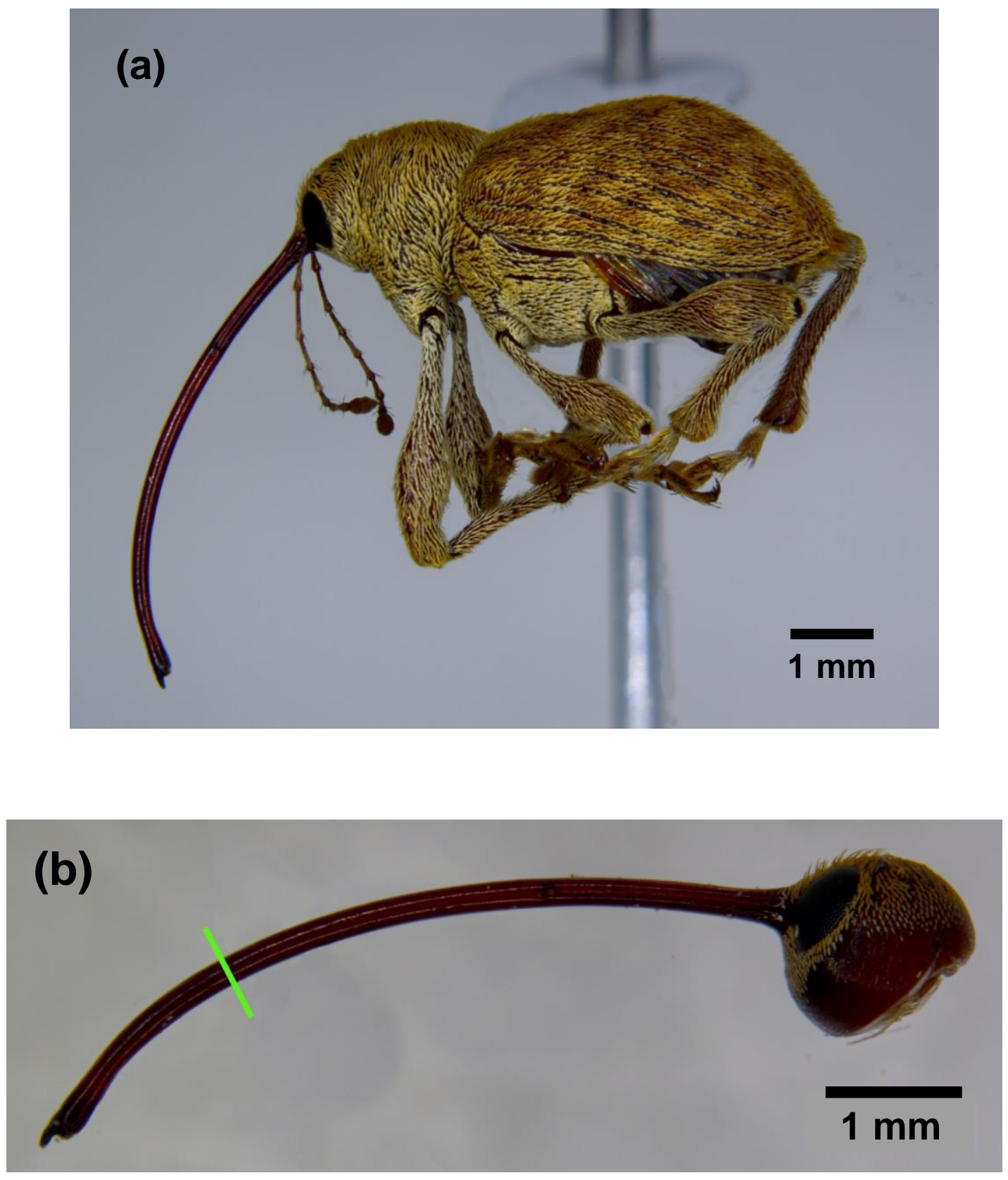

Fig. 1 

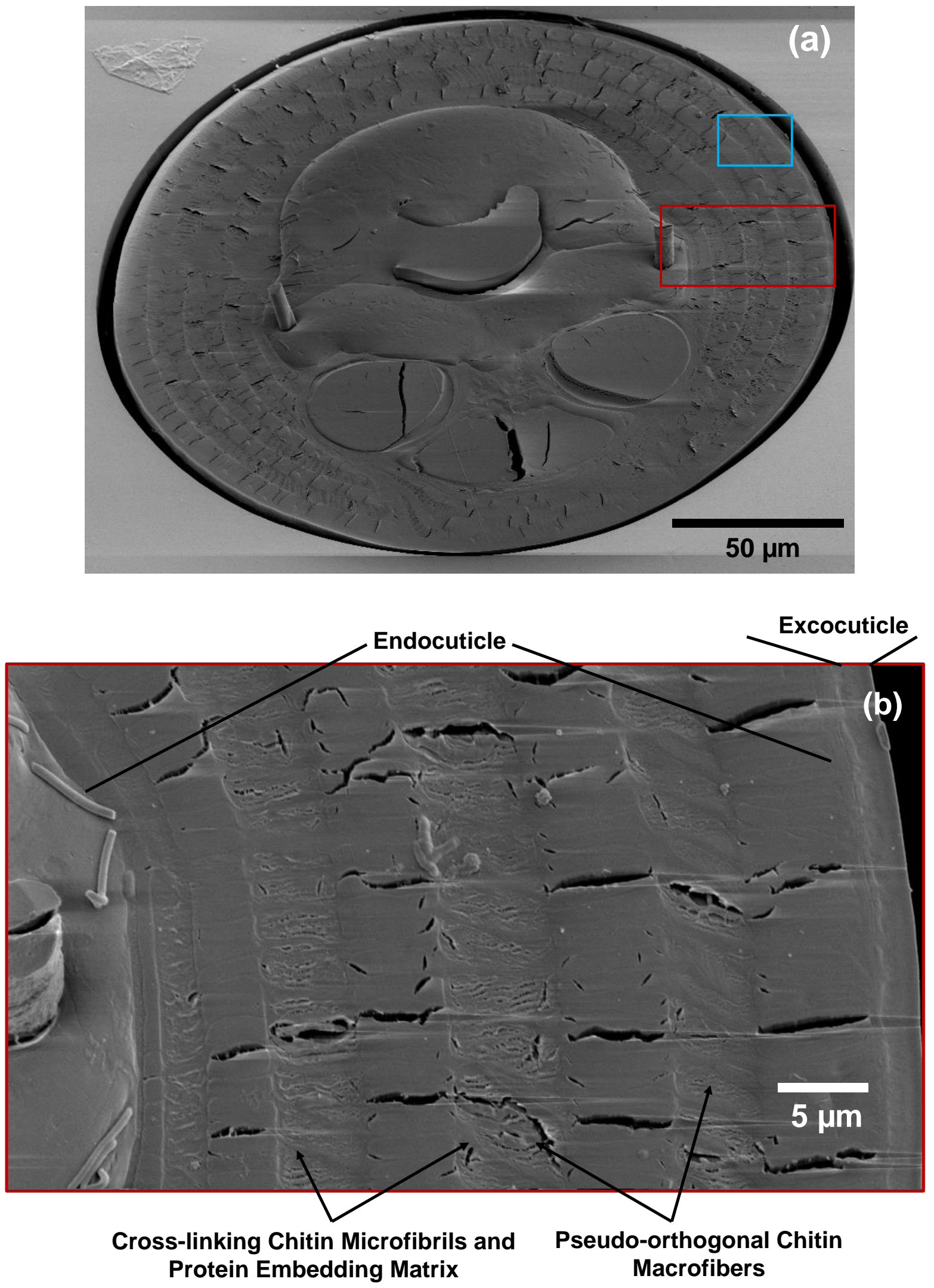


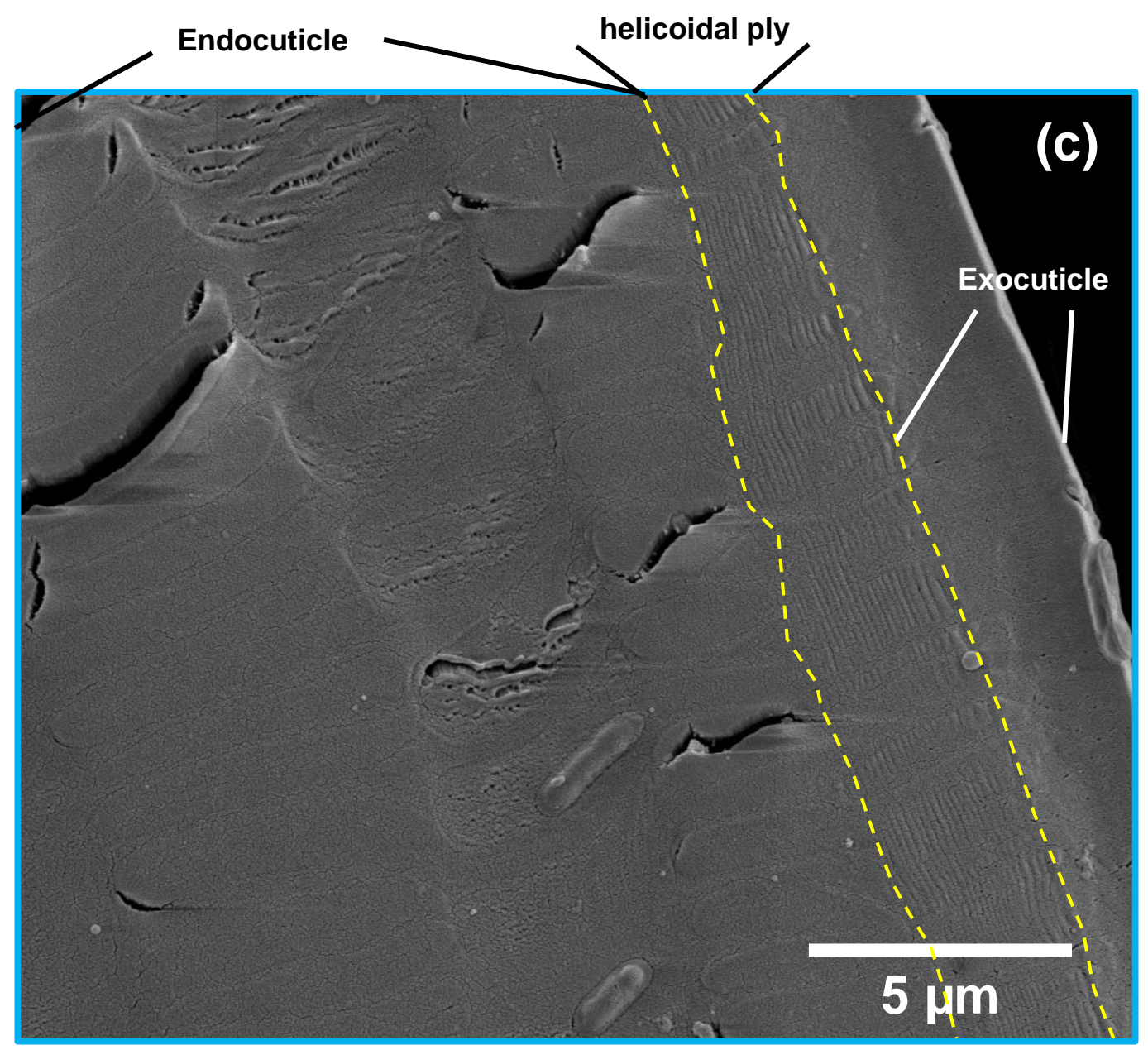

Fig. 2 


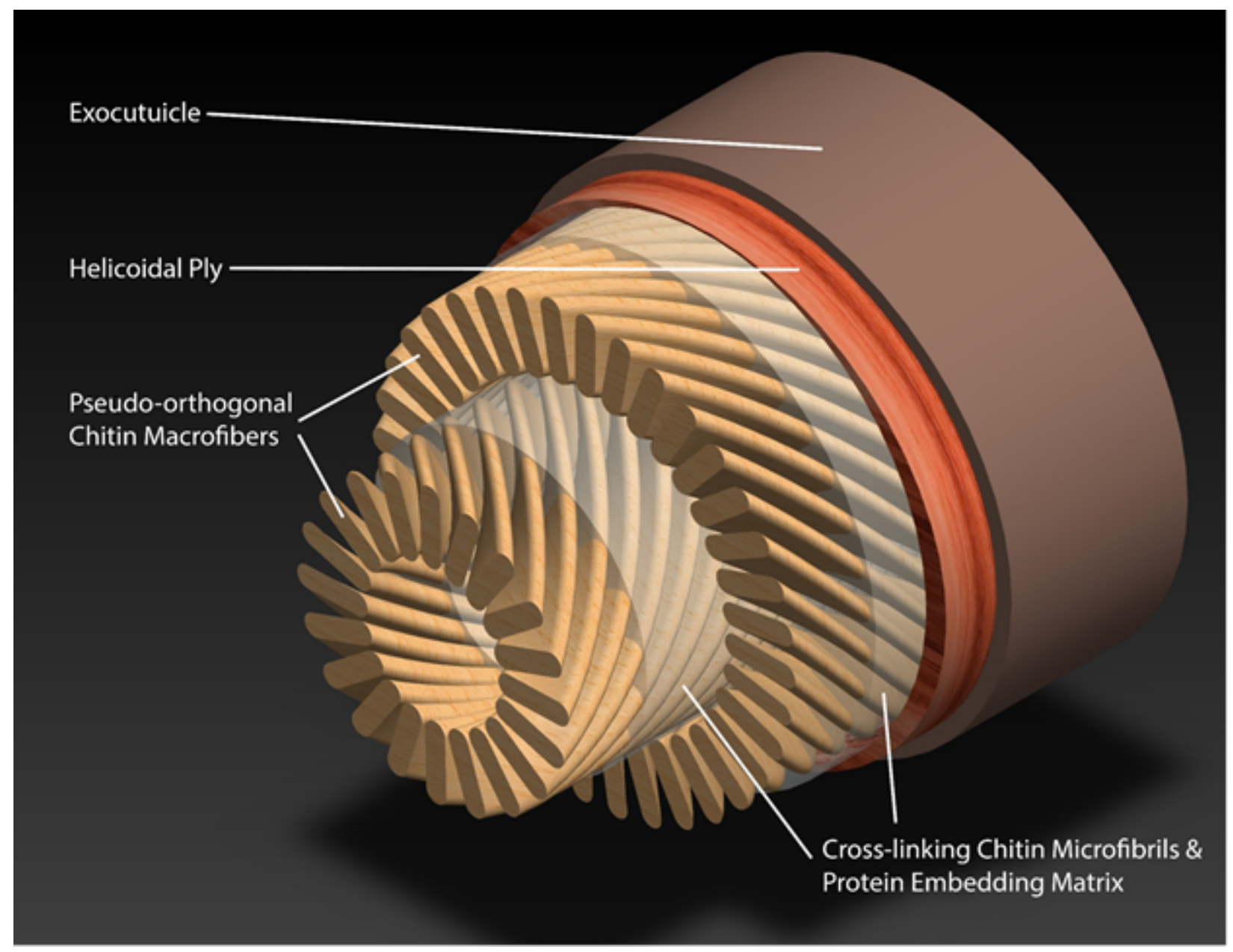

Fig. 3 


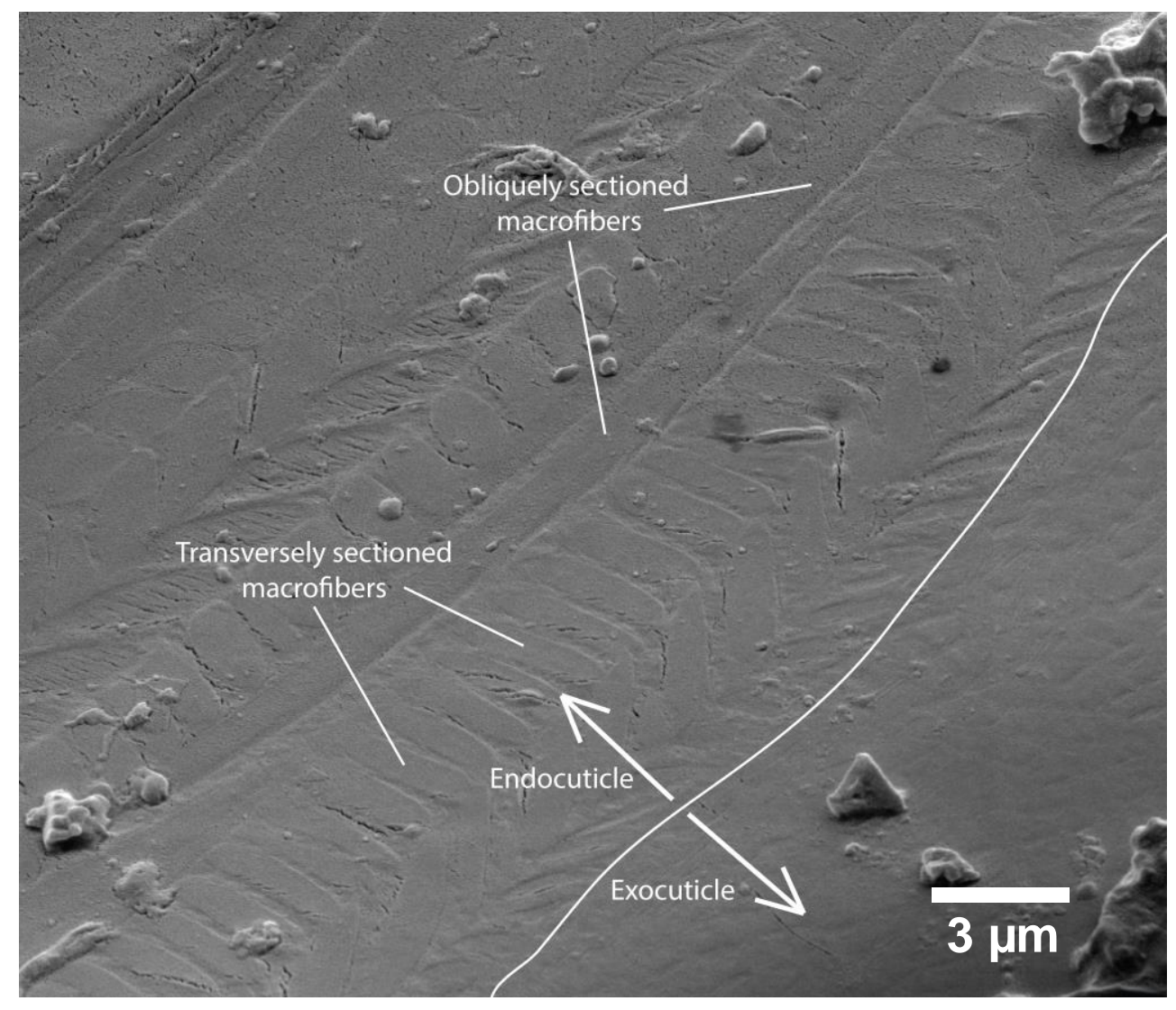

Fig. 4 


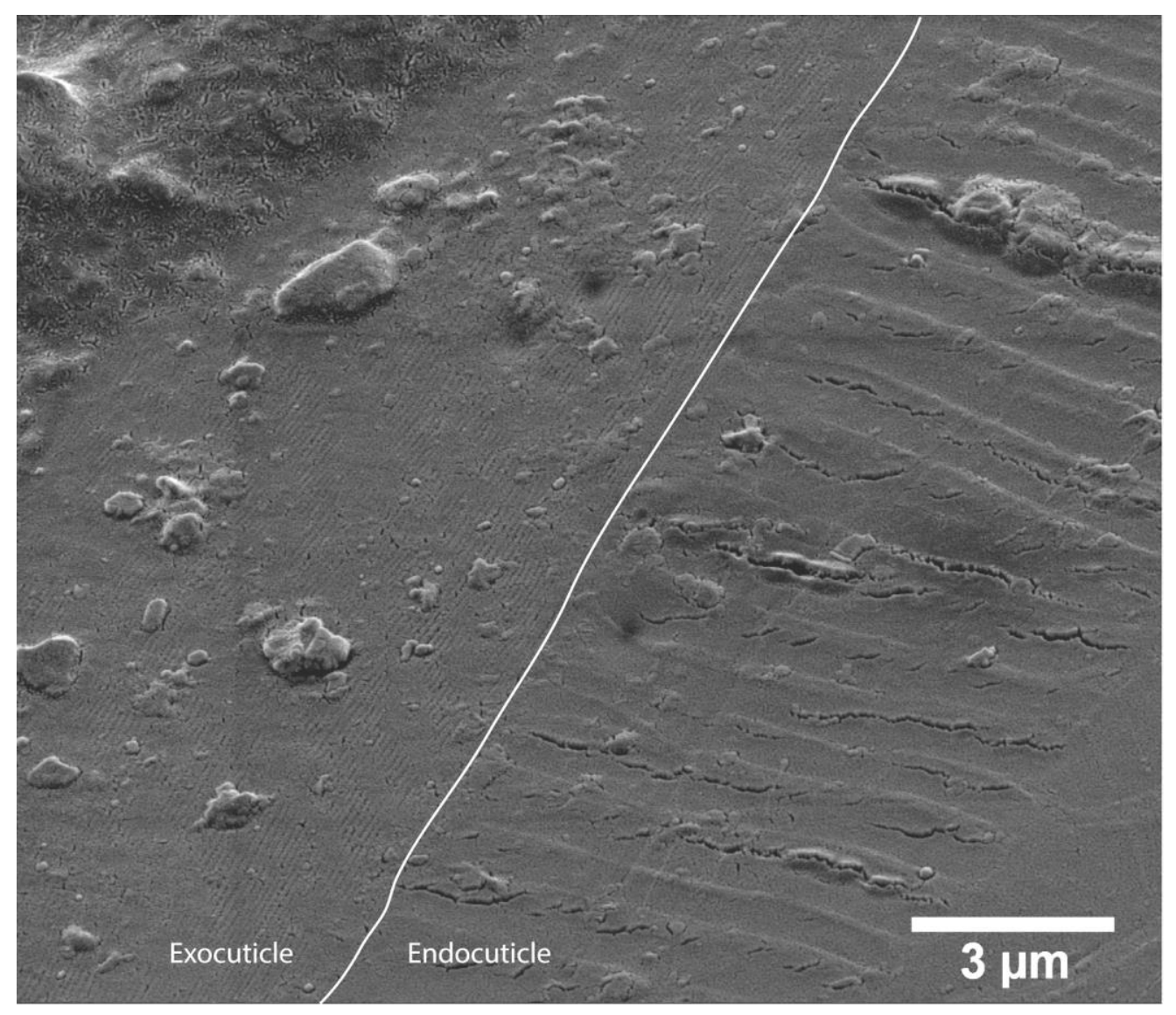

Fig. 5 


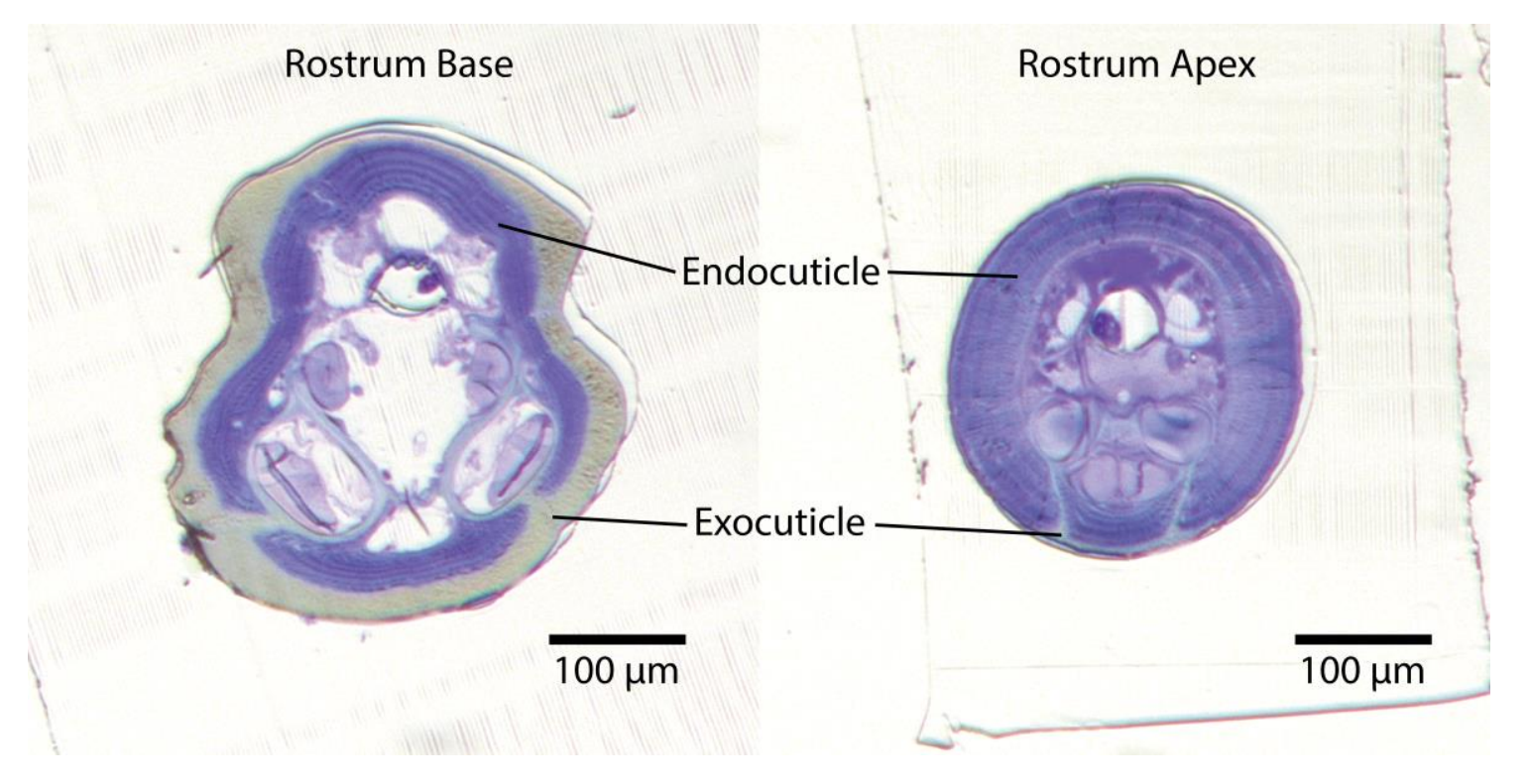

Fig. 6 

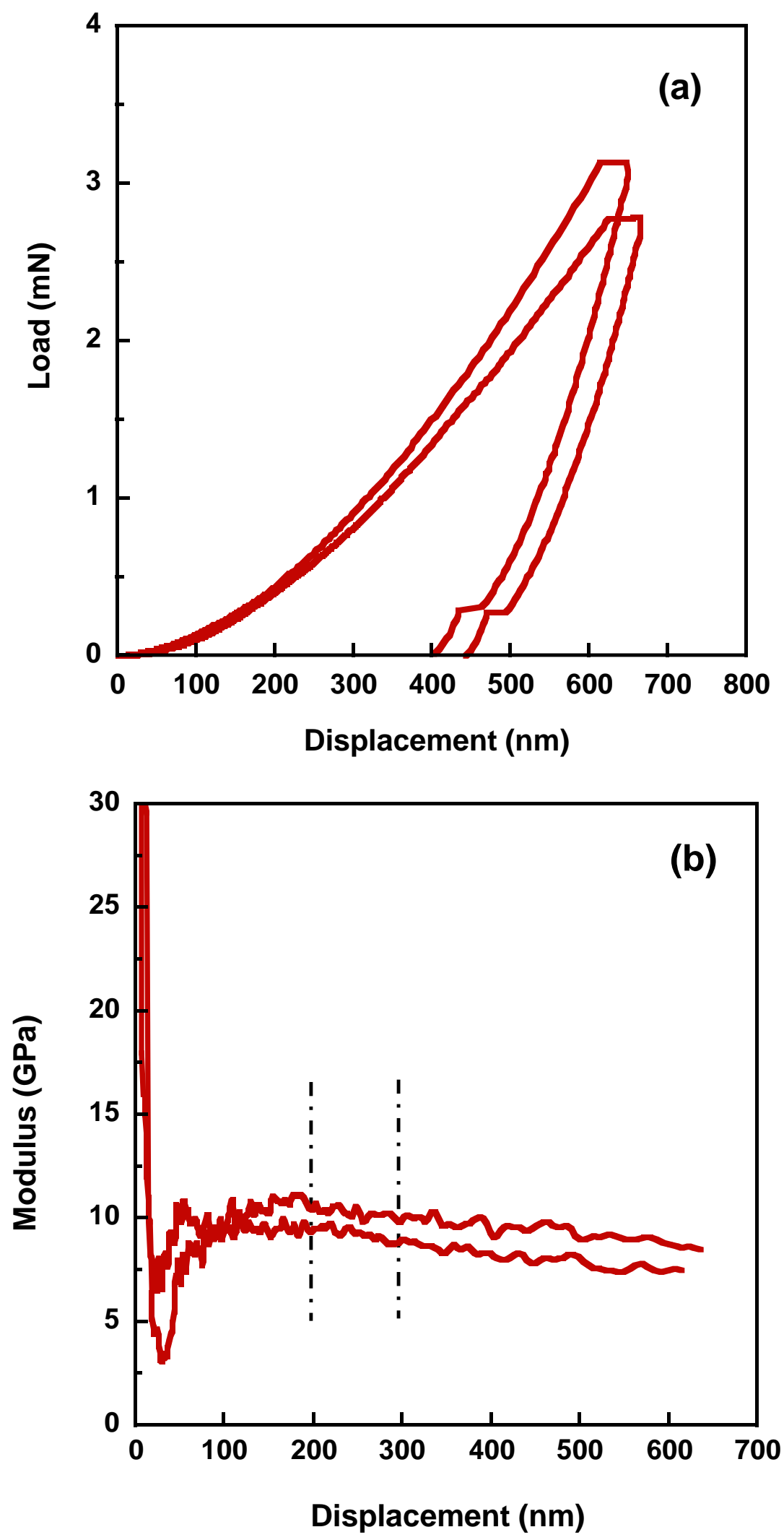


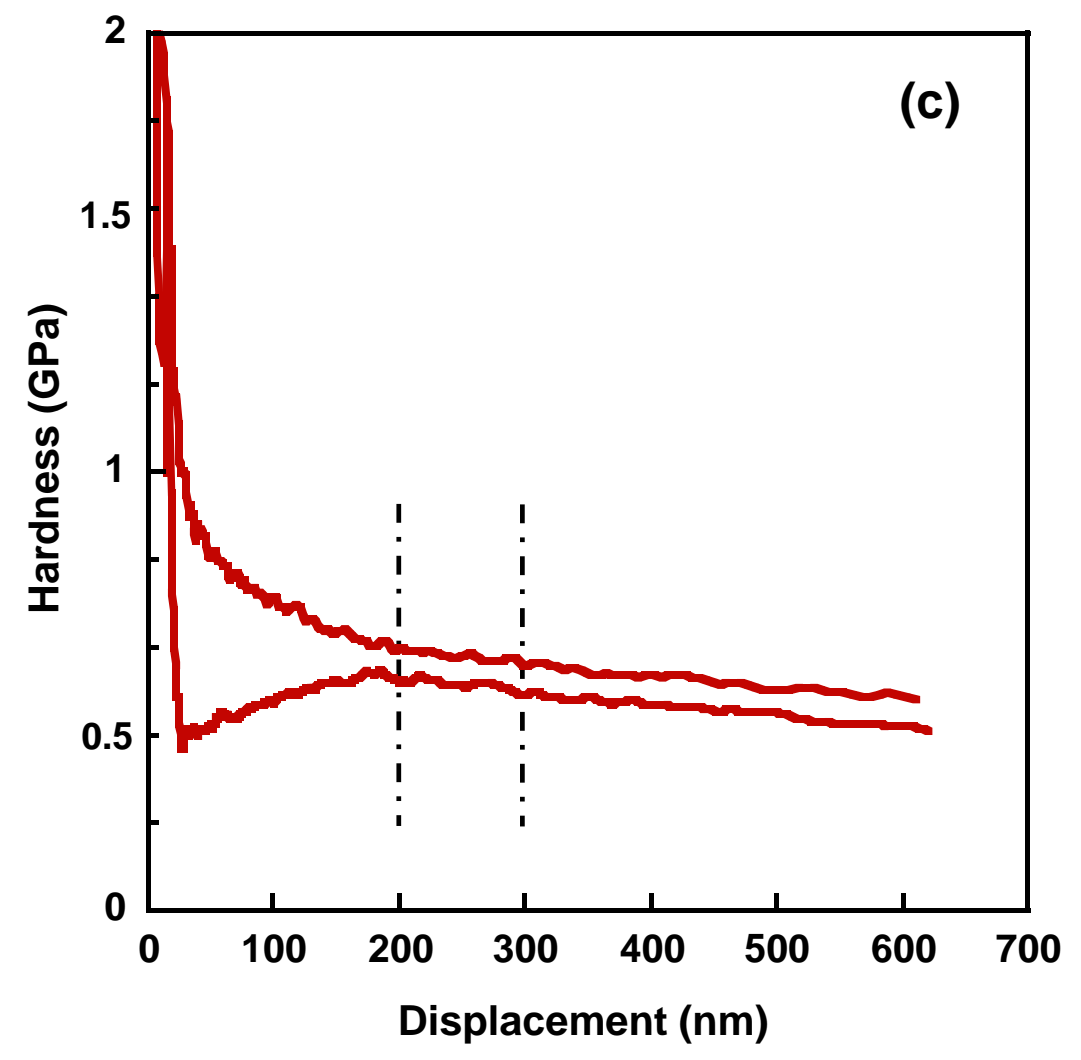

Fig. 7 


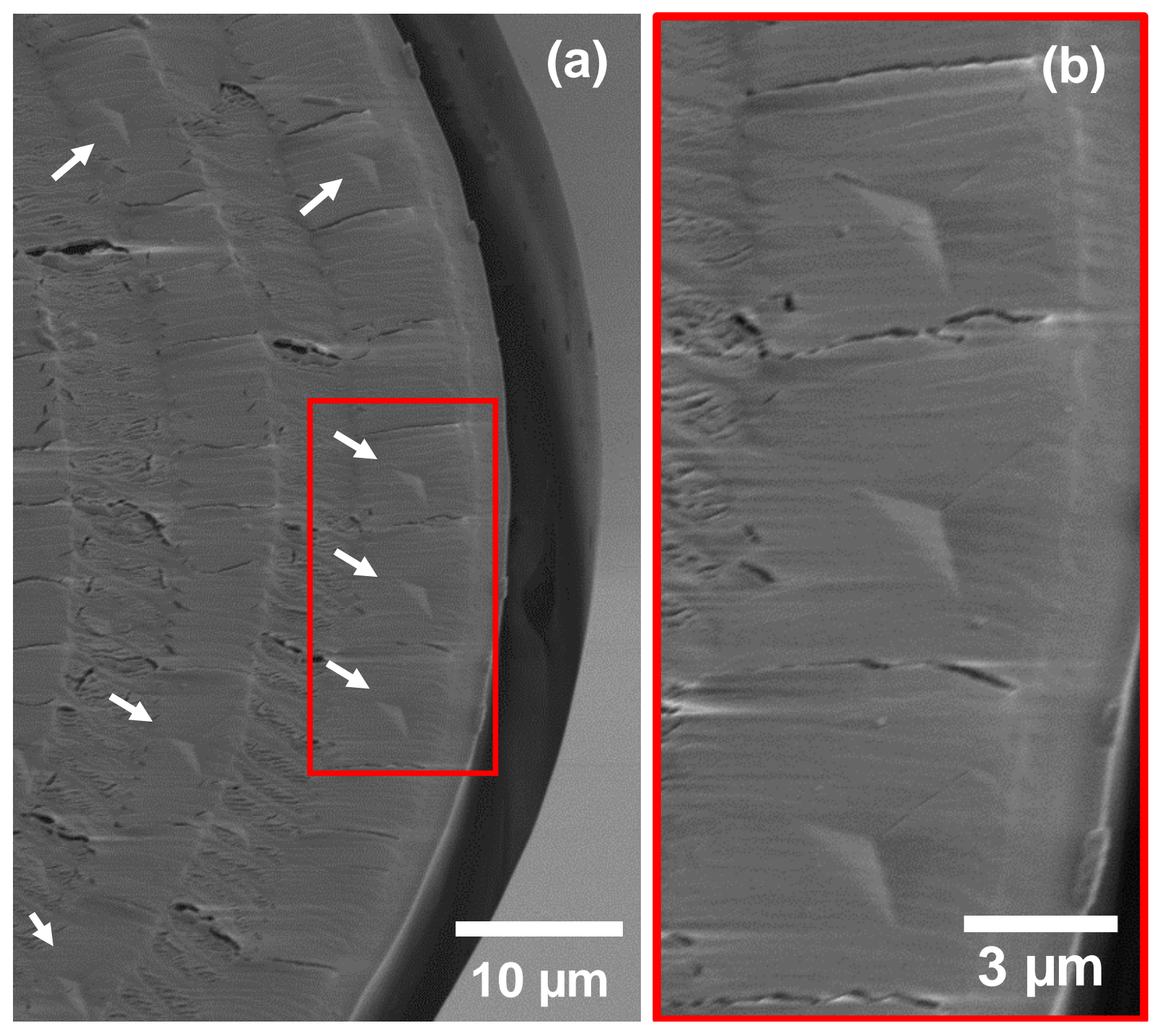

Fig. 8 